\title{
Thyroid gland volumes in premature infants using serial ultrasounds
}

\author{
Safdar S. Khan ${ }^{1} \cdot$ Irene Hong-McAtee ${ }^{2}$ Vesna Martich Kriss ${ }^{3} \cdot$ Scott Stevens ${ }^{1} \cdot$ Timothy Crawford $^{4} \cdot$ Mina Hanna $^{1}$. \\ Henrietta Bada ${ }^{1}$. Nirmala Desai ${ }^{1}$
}

Received: 21 December 2017 / Revised: 19 May 2018 / Accepted: 22 May 2018 / Published online: 30 July 2018

(c) Nature America, Inc., part of Springer Nature 2018

\begin{abstract}
Background There is a gap in knowledge about the postnatal growth of thyroid gland in preterm infants.

Objective To determine postnatal growth of thyroid gland in preterm infants.

Methods Thyroid gland volume was calculated in 57 prospectively enrolled preterm infants by measuring serial longitudinal, antero-posterior, and transverse dimensions of thyroid gland with ultrasound. Data were analyzed by using the Wilcoxon and independent $t$ test.

Results There was a significant correlation between thyroid volume (TV) and birthweight (BW) $(p=0.01)$, and between TV and gestational age $(p=0.02)$. However, unexpectedly, 12 infants had a decrease in TV between the first and second ultrasounds. Infants with late onset bacterial sepsis had lower TVs on their second ultrasounds than infants without sepsis.

Conclusions Thyroid ultrasound in preterm infants provides noninvasive and quick approach to determine TV and morphology. TV in preterm infants correlates positively with BW and gestational age. However, postnatal growth of thyroid gland is variable and may seemingly be affected by postnatal factors.
\end{abstract}

\section{Introduction}

The thyroid gland is a vital organ for normal neonatal growth and development. Congenital hypothyroidism $(\mathrm{CH})$ is a common disease in neonates, affecting approximately 1 per 4000 live births in the United States [1]. It is a preventable cause of intellectual and developmental disabilities; thus, early diagnosis and treatment are imperative [2,3]. An absent or ectopic gland is the most common cause of $\mathrm{CH}$ [4]. It is reported that $90 \%$ of cases of permanent $\mathrm{CH}$ have congenital thyroid agenesis, absence, or ectopic gland [5]. Historically, earlier detection through the introduction of newborn screening programs has essentially eradicated severe intellectual impairment due to $\mathrm{CH}$ in the developed world [6]. Several

Safdar S. Khan

ssk87@miami.edu

University of Kentucky, Lexington, KY, USA

Vanderbilt University, Nashville, TN, USA

3 Norton Children's Hospital, Louisville, KY, USA

4 Wright State University Boonshoft School of Medicine, Dayton, OH, USA patterns of thyroid dysfunction are seen in preterm infants. The most common pattern is transient hypothyroidism of prematurity, which is observed in up to $50 \%$ of infants born before 28 weeks [7]. Therefore, distinction of transient hypothyroidism from permanent hypothyroidism is crucial in vulnerable preterm infants. As dysmorphogenesis of thyroid gland is seen in the majority of infants with permanent hypothyroidism, measuring thyroid volume (TV) with ultrasonography may provide useful information in preterm infants. On anecdotal observations of thyroid ultrasounds (TUS) of preterm infants, we noticed that TV at birth increases with gestational age in neonatal population, and TV can reflect thyroid function. TV has been studied in full-term neonates and in fetuses $[8,9]$. However, to our understanding, there is a gap in knowledge about the postnatal growth of thyroid gland in preterm infants. Therefore, this is one of the first attempts to study postnatal thyroid growth using serial TUS in preterm infants. The specific aims of our study were to (1) measure TVs by serial TUS, and (2) to correlate serial TVs with body weight, postmenstrual age, cumulative radiation exposure, and late onset sepsis. 
Fig. 1 Flow diagram of the infants screened and enrolled
Time period: March $31^{\text {st }} 2008$-June $3^{\text {rd }} 2009$

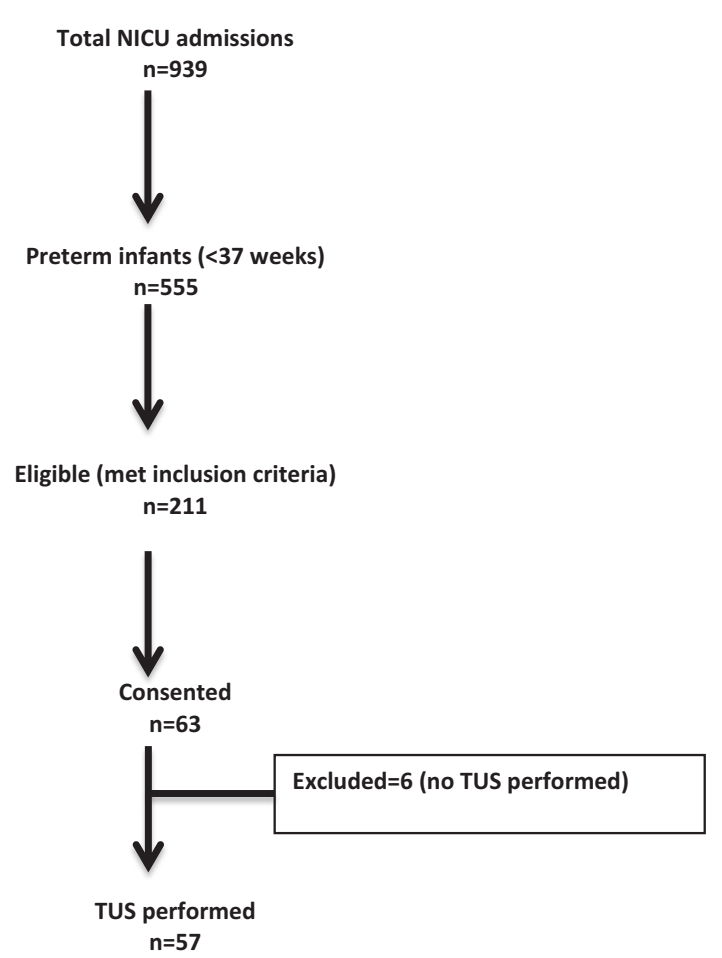

\section{Materials and methods}

\section{Study subjects}

This was a prospective observational study conducted at a single tertiary care center started after Institutional Review Board (IRB) approval. Infants were enrolled after informed parental consents were obtained. Inclusion criteria were: admission to the neonatal intensive care unit (NICU), gestational age at birth $<37$ weeks, clinical indication for ultrasound of any organ system, and parent(s) able to communicate in English.

Infants admitted to the NICU of Kentucky Children's Hospital (KCH) were screened for enrollment. After enrollment, thyroid ultrasound was performed only if there was a clinical indication to perform ultrasound for any other organ. As per standard of care, all preterm infants with gestation age $(\mathrm{GA})<32$ weeks and/or birthweight (BW) $<1500 \mathrm{~g}$ were screened for intraventricular hemorrhage (IVH) with head ultrasound (HUS). Based on the findings of initial HUS screen, a follow-up HUS was performed if indicated. At the time of HUS, the technician and/or the radiologist simultaneously performed ultrasound of the neck to measure TV. This allowed some consistency in the timing of first ultrasound. Thyroid ultrasound was also performed if an ultrasound was indicated for other clinical reasons in order to avoid multiple stimulations of preterm infants at different times.

\section{Measures}

TV was calculated based on longitudinal (sagittal), anteroposterior, and transverse (axial) dimensions, measured using multiplanar gray scale ultrasound with a $12 \mathrm{MHz}$ linear array transducer. To minimize interobserver variability, only two attending radiologists measured the thyroid dimensions and one ultrasound technician performed more than $50 \%$ of the ultrasounds. Infants were examined in the supine position with the neck hyperextended. To ensure that the probe was held in the same position each time, specific anatomical landmarks were used according to standard radiological guidelines. Using a linear probe with prewarmed coupling gel, transverse and longitudinal sections of both lobes of the thyroid gland were scanned. For measurement of thyroid length, the probe was placed longitudinally in the midline of the neck to obtain sagittal views of the larynx; the probe was then moved obliquely to find the maximum length of the thyroid gland, just medial to the carotid vessels. The transverse views were obtained by using the trachea and carotid vessels as landmarks. The TV was calculated using the formula: TV $=$ length $\times$ breadth $\times$ depth $\times \mathrm{Pi} / 6$ [8].

Total thyroxine (T4) and thyroid-stimulating hormone (TSH) levels were collected by heel-stick and measured per standard of care as part of the Kentucky state newborn screen. 
Table 1 Demographics and characteristics of the infants enrolled

\begin{tabular}{ll}
\hline & $N=57$ \\
\hline Gestational age, mean (SD) & 28.9 weeks (3.28) \\
Birthweight, mean (SD) & $1181.7 \mathrm{~g}(471.5)$ \\
Male (\%) & $31(54.4)$ \\
SGA (\%) & $5(8.8)$ \\
Normal TSH (\%) & $57(100)$ \\
Abnormal T4 (\%) & $14(24.6)$ \\
$N$ requiring treatment (\%) & $2(3)$ \\
Maternal thyroid Rx & 0 \\
Maternal steroids (\%) & $49(86)$ \\
Race ( $n, \%)$ & \\
$\quad$ White & $55(96)$ \\
$\quad$ African American & $2(4)$ \\
Delivery mode, C-section (\%) & $44(77)$ \\
1 min Apgar, mean (SD) & $5.3(2.3)$ \\
5 min Apgar, mean (SD) & $6.8(2.1)$ \\
$\mathrm{O}_{2}$ requirement (\%) & $52(91)$ \\
Ventilator days mean (SD) & $11.7(16.8)$ \\
RDS (\%) & $44(75.4)$ \\
PDA (\%) & $27(47.4)$ \\
NEC (\%) & $4(7)$ \\
Sepsis (\%) & $15(26.3)$ \\
BPD (\%) & $11(19.3)$ \\
Length of stay, mean (SD) & $60.4(36.4)$ \\
\hline Abbreviato & \\
\hline &
\end{tabular}

Abbreviations: $S D$ standard deviation, $S G A$ small for gestational age, TSH thyroid-stimulating hormone, HFOV high frequency oscillation ventilation, $N C$ nasal cannula, $R D S$ respiratory distress syndrome, $P D A$ patent ductus arteriosus, $N E C$ necrotizing enterocolitis, $B P D$ bronchopulmonary dysplasia.

Electronic Medical Records were used to collect information regarding demographics and characteristics, including GA, BW, physical examination parameters on admission, gender, Apgar scores, maternal prenatal care, antenatal steroids, mode of delivery, respiratory distress syndrome, oxygen requirement, need for respiratory support, neonatal sepsis, necrotizing enterocolitis, patent ductus arteriosus, gastrointestinal perforation, retinopathy of prematurity, and length of stay (LOS). Sepsis was defined as treatment with antibiotics of a culture positive organism including CSF and blood. Postnatal growth curve of thyroid gland was also developed and compared with fetal growth curve [10].

\section{Statistical analysis}

Analyses were carried out using SAS v 9.2 software (SAS Institute, Cary, NC). Group comparisons were analyzed using the Wilcoxon and independent $t$ test. A $p<0.05$ was considered statistically significant.

\section{Results}

During the study period between March 31, 2008 and June 3, 2009, 939 infants were admitted to the NICU at $\mathrm{KCH}$. There were 555 infants $<37$ weeks and 211 infants met inclusion criteria for enrollment. In total, 63 infants were enrolled. Six infants were excluded as no thyroid ultrasound (TUS) was performed. Finally, analysis was performed on 57 infants. Figure 1 shows the flow diagram of study enrollment.

Table 1 shows the demographics and clinical characteristics of infants enrolled including mean gestational age (GA) $(28.9 \pm 3.28)$, birthweight $(1181.7 \mathrm{~g} \pm 471.5)$, clinical parameters, and LOS.

Table 2 shows the mean chronological ages at which TUS were performed as well as TVs. In 57 enrolled infants, the first TUS was performed at mean chronological age of 13 days. Mean thyroid width was $9 \mathrm{~mm} \pm$ 1.8 standard deviation (SD) and mean TV was $185 \mathrm{~mm}^{3} \pm$ 100 SD on first TUS. Of the 57 infants, 38 infants had second TUS at mean chronological age of 38 days. Mean thyroid width was $9.4 \mathrm{~mm} \pm 1.6 \mathrm{SD}$ and mean TV was $198 \mathrm{~mm}^{3} \pm 76 \mathrm{SD}$ on second TUS. Of the 38 infants, 15 infants had third TUS at mean chronological age of 64 days. Mean thyroid width was $10.2 \mathrm{~mm} \pm 1.5$ SD and mean TV was $237 \mathrm{~mm}^{3} \pm 78 \mathrm{SD}$ on third TUS. Of the 15 infants, 4 infants had fourth TUS at mean chronological age of 95 days. Mean thyroid width was 11 $\mathrm{mm} \pm 1.5 \mathrm{SD}$ and mean TV was $365 \mathrm{~mm}^{3} \pm 226 \mathrm{SD}$ on fourth TUS.

There was a significant correlation between thyroid volume and birthweight $(p=0.01)$ as well as between thyroid volume and gestational age $(p=0.02)$ (Fig. 2).

Figure 3 shows individual profiles with an average trend line of thyroid volumes versus postmenstrual age. It was noticed that there was an overall positive correlation between thyroid volume and postmenstrual age as well. However, 12 of 38 (31\%) infants had decrease in thyroid volume between the first and second TUS. This decrease was not related to postmenstrual age, or cumulative radiation exposure.

Figure 4 compares thyroid volume between infants with the diagnosis of sepsis versus no sepsis. There was a trend toward a lower thyroid volume on the second ultrasound in the sepsis group $(p=0.27)$. In infants without sepsis, there was a significant change in thyroid volume between first and second ultrasounds (mean difference $=110.21 \pm 79.98$, $p=0.01)$.

In order to evaluate the postnatal trend of growth in preterm infants, Achiron's published thyroid widths of fetuses (1998) [9] were compared with thyroid widths of infants with similar gestational ages. Initial thyroid widths of preterm infants were lower and continued to be lower 
Table 2 Mean chronological age, weight, thyroid volumes, and radiation exposure

\begin{tabular}{lllll}
\hline Serial TUS \# & First & Second & Third & Fourth \\
\hline$N$ & 57 & 38 & 15 & 4 \\
Chronologic age (days), average (age range) & $13(1-56)$ & $37(7-82)$ & $64(37-88)$ & $95(76-129)$ \\
Post menstrual age in weeks, mean $( \pm$ SD) & $31.3(3.6)$ & $33.0(3.0)$ & $35.6(2.6)$ & $38.9(5.0)$ \\
Weight in grams, mean $( \pm$ SD) & $1340(611)$ & $1554(514)$ & $2041(480)$ & $2831(794)$ \\
Thyroid width in mm, mean $( \pm \mathrm{SD})$ & $9.0(1.8)$ & $9.4(1.6)$ & $10.2(1.5)$ & $11.0(3.4)$ \\
Thyroid volume in mm ${ }^{3}$, mean $( \pm \mathrm{SD})$ & $185(100)$ & $198(76)$ & $237(78)$ & $365(226)$ \\
Number of X-rays prior to TUS, mean $( \pm \mathrm{SD})$ & $12.1(7.9)$ & $21.7(9.3)$ & $32.7(9.1)$ & $33.7(6.8)$ \\
\hline
\end{tabular}

Initial thyroid volume increases with birthweight $(\mathrm{p}=0.002)$

\begin{tabular}{|l|c|c|c|}
\hline $\begin{array}{l}\text { Birth weight } \\
\text { group } \\
\text { (grams) }\end{array}$ & $N(\%)$ & $\begin{array}{c}\text { Mean } \\
\text { thyroid } \\
\text { volume } \\
(\mathrm{SE})\end{array}$ & p value \\
\hline$<1000$ & $24(42.1)$ & $161(90)$ & \\
\hline $1000-1500$ & $18(31.6)$ & $182(64)$ & \\
\hline $1500-2000$ & $12(21.1)$ & $205(70)$ & \\
\hline$>2000$ & $3(5.3)$ & $306(208)$ & \\
\hline & & & 0.01 \\
\hline
\end{tabular}

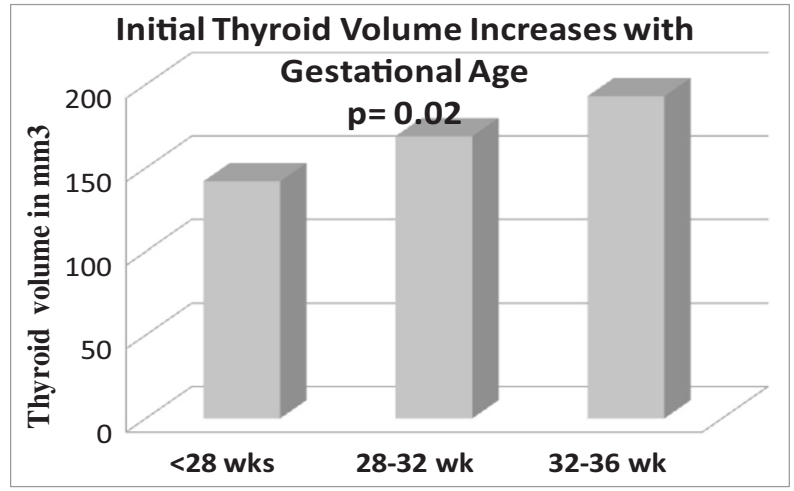

Fig. 2 Association of thyroid volume with birthweight and gestational age

compared to intrauterine fetal thyroid measurements as shown in Fig. 5.

\section{Discussion}

Data on TVs in fetuses as well as normative data in term infants, measured by ultrasonography, have been published in previous studies $[8,9]$. Our study is an attempt to fill the

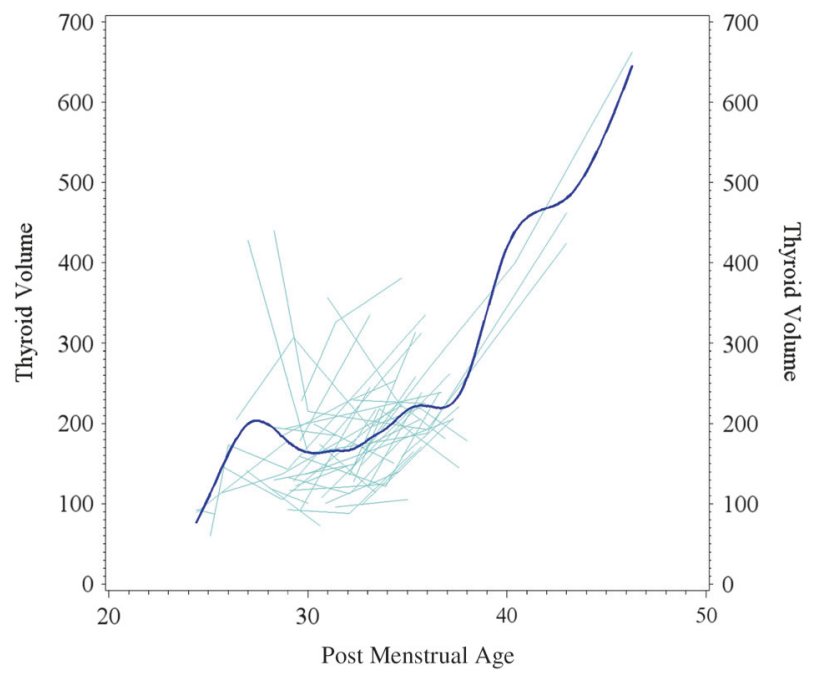

Fig. 3 Postmenstrual age and thyroid volume

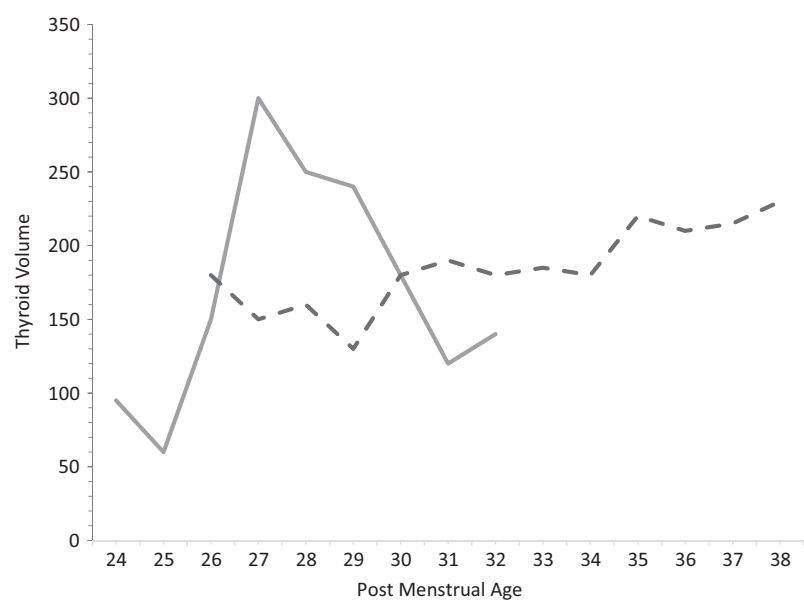

Fig. 4 Comparison of thyroid volumes in septic versus nonseptic infants

gap in knowledge about postnatal growth of thyroid gland in preterm infants.

Thyroid ultrasonography is a noninvasive diagnostic imaging technique, which can be used to measure TV in preterm infants. As the study shows, thyroid ultrasound can 


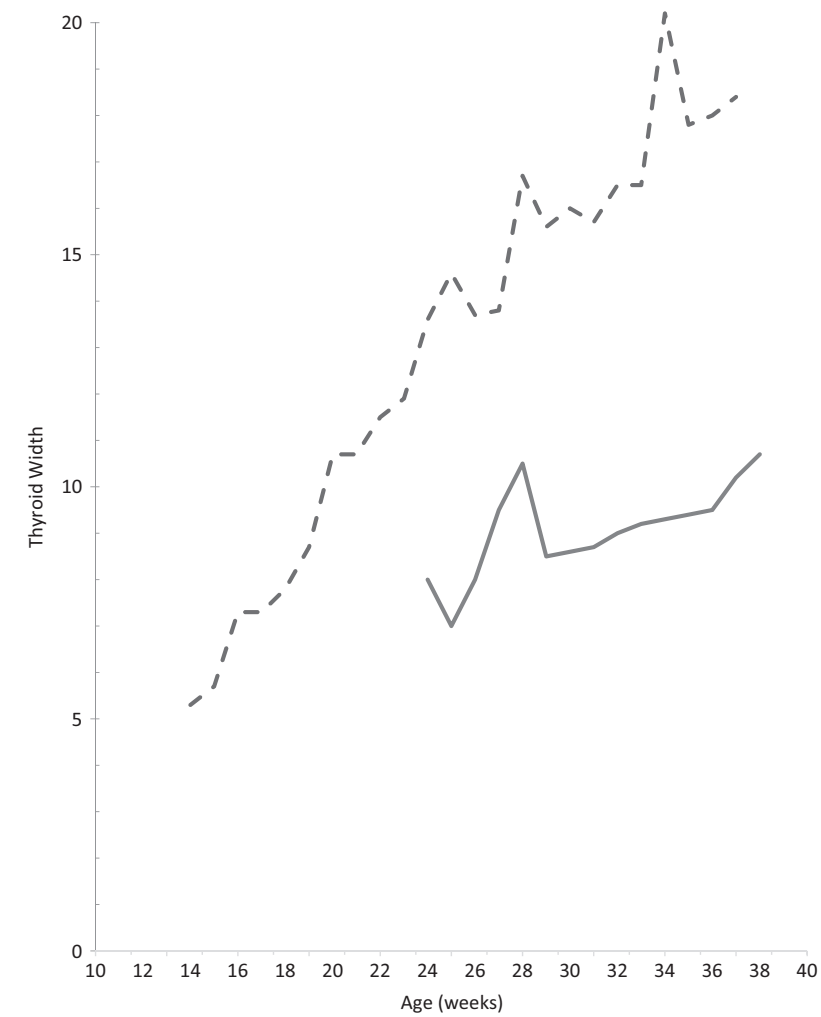

Fig. 5 Postnatal growth curve of thyroid gland compared to fetal curve

provide an immediate approach to determine whether there is thyroid tissue in the correct position pending newborn screen results. Establishing the thyroid gland is small, normal, or enlarged is important. There were limited data on TVs and postnatal growth in preterm infants.

Ares et al. [11] determined TVs by ultrasonography in 30 preterm infants (27-36 weeks' gestation) born in Madrid. Despite different racial makeup of target population and higher percentage of low BW infants $(<1500 \mathrm{~g}=73.7 \%)$, results of our study were consistent with the Spanish study, and demonstrated that TV significantly increased with postnatal age and was very well correlated with body weight. However, interestingly, on subanalysis of the infants with late onset sepsis, initial TVs were significantly lower in infants with bacterial sepsis warranting further studies to ascertain association. On comparison of Achiron's published thyroid widths of fetuses [9] with postnatal widths of our study, it was noticed that measurements of thyroid widths of premature infants were lower initially, and continued to be lower compared to intrauterine measurements. However, this could be related to variation in measurements (intrauterine versus postnatal) as well as use of historical data as controls. Further studies are needed to elucidate differences in fetal and neonatal thyroid growths by comparing with similar controls.

Although there was a significant association between TV and weight gain $(p=0.01), 12$ out of 38 infants had a decrease in TV rather than an increase, between first and second TUS. This decrease in TV was not related to postmenstrual age, protein intake, or cumulative radiation exposure, suggesting postnatal growth variability of thyroid gland in preterm infants as compared to term infants. Further studies are needed to determine whether other measures of severity of illness are associated with changes in TV.

One of the strengths of our study is that compared to previous studies, two independent observers viewed the scans to minimize interobserver variability. There are a number of limitations of the study. First, even though, to our knowledge, this is the largest sample of preterm infants where TV was measured with serial TUS, it is still a small sample size to establish normative data in preterm infants. Second, thyroid function tests were not measured simultaneously with TUS; rather, T4 and TSH were determined from newborn screen results. Therefore, the study does not provide association of TV with thyroid function tests. Third, maternal thyroid function tests were not measured as well; rather, obstetric admission notes were used to determine whether or not there was a history of maternal thyroid disease. Nonetheless, this study adds to the literature by providing TVs in preterm infants by using a simple and noninvasive technique, and provides basis for further studies. A recent study found that thyroid dysfunction is common among premature infants, with the majority of cases with thyroid dysfunction had $\mathrm{CH}$, with delayed TSH elevation, which was not detected by newborn screening [12]. Furthermore, TV measurements in preterm infants can facilitate comparison of results with other thyroid studies, where TV was measured by using ultrasonography.

\section{Summary and conclusion}

Thyroid ultrasound in preterm infants provides a noninvasive and quick approach to determine TV and morphology. TV in preterm infants correlates positively with BW and gestational age. However, postnatal growth of thyroid gland is variable and seemingly affected by postnatal factors. Further studies are needed to elucidate whether measures of severity of illness are associated with changes in TV.

Acknowledgements The project described was supported by Children's Miracle Network (CMN) grant awarded to Kentucky Children's Hospital. We thank our research nurses Vicky Whitehead and Deb Grider for their assistance. We thank parents, nurses and doctors at Kentucky Children's hospital for their contribution in completion of this study.

Funding The project described was supported by the Children's Miracle Network (CMN) grant awarded to Kentucky Children's Hospital. 


\section{Compliance with ethical standards}

Conflict of interest The authors declare that they have no conflict of interest.

\section{References}

1. Delange F. Screening for congenital hypothyroidism used as an indicator of the degree of iodine deficiency and of its control. Thyroid. 1998;8:1185-92.

2. Zhan JY, Qin YF, Zhao ZY. Neonatal screening for congenital hypothyroidism and phenylketonuria in China. World J Pediatr. 2009;5:136-9.

3. Macchia PE, De Felice M, Di Lauro R. Molecular genetics of congenital hypothyroidism. Curr Opin Genet Dev. 1999;9:289-94.

4. Gaudino R, Garel C, Czernichow P, Léger J. Proportion of various types of thyroid disorders among newborns with congenital hypothyroidism and normally located gland: a regional cohort study. Clin Endocrinol (Oxf). 2005;62:444-8.
5. Yao D, He X, Yang RL, Jiang GP, Xu YH, Zou CC, et al. Sonographic measurement of thyroid volumes in healthy Chinese infants aged 0-12 months. J Ultrasound Med. 2011;30:895-8.

6. Wassner AJ, Brown RS. Hypothyroidism in the newborn period. Curr Opin Endocrinol Diabetes Obes. 2013;20:449-54.

7. La Gamma EF, Paneth N. Clinical importance of hypothyroxinemia in the preterm infant and a discussion of treatment concerns. Curr Opin Pediatr. 2012;24:172-80.

8. Perry RJ, Hollman AS, Wood AM, Donaldson MD. Ultrasound of the thyroid gland in the newborn: normative data. Arch Dis Child Fetal Neonatal Ed. 2002;87:F209-11.

9. Achiron R, Rotstein Z, Lipitz S, Karasik A, Seidman DS. The development of the foetal thyroid: in utero ultrasonographic measurements. Clin Endocrinol (Oxf). 1998;48:259-64.

10. Ho SS, Metreweli C. Normal fetal thyroid volume. Ultrasound Obstet Gynecol. 1998;11:118-22.

11. Ares S, Pastor I, Quero J, Morreale de Escobar G. Thyroid gland volume as measured by ultrasonography in preterm infants. Acta Paediatr. 1995;84:58-62.

12. Kaluarachchi DC, Colaizy TT, Pesce LM, Tansey M, Klein JM. Congenital hypothyroidism with delayed thyroid-stimulating hormone elevation in premature infants born at less than 30 weeks gestation. J Perinatol. 2017;37:277-82. 